Research Article

\title{
Certain Bounds of Regularity of Elimination Ideals on Operations of Graphs
}

\author{
Zongming Lv (D), ${ }^{1}$ Muhammad Junaid Ali Junjua, ${ }^{2}$ Muhammad Tajammal Tahir, \\ and Khurram Shabbir $\mathbb{D}^{2}$ \\ ${ }^{1}$ School of Information and Finance, Xuancheng Vocational \& Technical College, Xuancheng, 242000, AnHui, China
${ }^{2}$ Department of Mathematics, G. C. University, Lahore, Pakistan
}

Correspondence should be addressed to Zongming Lv; lzm_1234562021@163.com and Khurram Shabbir; dr.khurramshabbir@ gcu.edu.pk

Received 2 September 2021; Revised 30 September 2021; Accepted 4 October 2021; Published 29 October 2021

Academic Editor: Muhammad Faisal Nadeem

Copyright (c) 2021 Zongming Lv et al. This is an open access article distributed under the Creative Commons Attribution License, which permits unrestricted use, distribution, and reproduction in any medium, provided the original work is properly cited.

Elimination ideals are regarded as a special type of Borel type ideals, obtained from degree sequence of a graph, introduced by Anwar and Khalid. In this paper, we compute graphical degree stabilities of $K_{n} \vee C_{m}$ and $K_{n} * C_{m}$ by using the DVE method. We further compute sharp upper bound for Castelnuovo-Mumford regularity of elimination ideals associated to these families of graphs.

\section{Introduction}

Let $S=k\left[x_{1}, \ldots, x_{n}\right], n \geq 2$ be a polynomial ring in $n$ variables over an infinite field $k$. Bayer and Stillman (see [1]) noted that a monomial ideal $I \subset S$ is of Borel type, if it satisfies the following condition:

$$
\left(I: x_{j}^{\infty}\right)=\left(I:\left(x_{1}, \ldots, x_{j}\right)^{\infty}\right), \quad \text { for all } 1 \leq j \leq n .
$$

The Castelnuovo-Mumford regularity (or simply regularity) gives an estimate of the complexity of computing Grö bner bases. The regularity of an ideal $I$ is defined as $\operatorname{reg}(I)=\left\{\max j: \beta_{i, i+j}(I) \neq 0\right\}$, where $\beta_{i j}$ 's are the graded Betti numbers of ideal $I$. The regularity of monomial ideals of Borel type was extensively studied (see for instance [2-4]).

The study of degree sequence started in 1981 by Bollobas [5]. Tyshkevich et al. in [6] established a correspondence between degree sequence of a graph and some structural properties of this graph. Havel-Hakimi criterion (see [7]) states that a sequence $\left(d_{1}, d_{2}, \ldots, d_{n}\right)$ of non-negative integers such that $d_{1} \geq d_{2} \geq \cdots \geq d_{n}$ is graphic if and only if the sequence $\left(d_{2}-1, \ldots, d_{d_{1}+1}-1, d_{d_{1}+2}, \ldots, d_{n}\right)$ is graphic. Given a non-negative sequence $\left(d_{1}, d_{2}, \ldots, d_{n}\right)$, this sequence will be called realizable if it is a degree sequence of any graph.
Throughout this paper, we assume $H$ to be a simple, finite, and connected graph and label its vertices according to the degree sequence in descending order. In figures, we identify the vertex $v_{i}$ of $H$ with the positive integer $i$. Further, we identify a vertex $v_{i}$ of a graph $H$ with variable $x_{i}$ of the polynomial ring $S=k\left[x_{1}, \ldots, x_{n}\right]$. Recently, Anwar and Khalid in [2] introduced a new class of monomial ideals, namely, elimination ideals $I_{D}(H)$, associated to a graph $H$. They showed that elimination ideals are the monomial ideals of Borel type. They gave the description of graphical degree stability of graph $H$ denoted by $\operatorname{Stab}_{d}(H)$, a combinatorial measure associated to $H$. They gave a systematic procedure to compute the graphical degree stability, namely, dominating vertex elimination method (DVE method). In $[2,8]$, the authors computed the upper bounds of the Castelnuovo-Mumford regularity of elimination ideals associated to some families of graphs [9-11].

Motivated by the studies in $[2,8]$, we further extend this study for join and corona product of complete and cyclic graphs. We compute the graphical degree stabilities of join and corona product of above graphs by using the dominating vertex elimination method (see Lemmas 1 and 2). We give a description on computing the sharp upper bounds for 
the regularity of elimination ideals associated to these families of graphs respectively (see Theorems 1 and 2).

\section{Preliminaries}

Definition 1. Let $H$ be a simple, finite, and connected graph with vertex set $V(H)$. The degree of a vertex $v \in V(H)$, denoted by $d(v)$, is the number of edges at $v$.

A vertex $v_{i}$ of a graph $H$ is called dominating vertex, if $\operatorname{deg}\left(v_{i}\right) \geq \operatorname{deg}\left(v_{j}\right)$ for all $v_{j} \in V(H)$ with $i \neq j$. The set of all dominating vertices of a graph $H$ is called dominating set and is denoted by $D(H)$. A graph $H$ is called scattered graph if it has at least one vertex with degree zero; otherwise, it is called non-scattered graph.

Here, we introduce different operations on graphs.

Definition 2. Let $G$ and $H$ be two graphs with mutually disjoint vertex sets $V(G)=\left\{u_{1}, u_{2}, \ldots, u_{n}\right\}$ and $V(H)=\left\{v_{1}, v_{2}, \ldots, v_{m}\right\}$. A graph denoted by $G \vee H$ is called the join of $G$ and $H$ if $V(G \vee H)=V(G) \cup V(H)$ and $E(G \vee H)=E(G) \cup E(H) \cup\left\{u_{i} v_{j} \mid u_{i} \in V(G), v_{j} \in V(H)\right\}$.

Definition 3. The corona product of two graphs $G$ and $H$ is the graph obtained by taking one copy of $G$ and $|V(G)|$ disjoint copies of $H$ and then joining the $j^{\text {th }}$ vertex of $G$ to every vertex in the $j^{\text {th }}$ copy of $H$. We denote this graph as $G * H$. Corona product of two graphs $G$ and $H$ with $n$ and $m$ vertices, respectively, has $n+n m$ vertices.

Definition 4. Let $H_{i}$ be a graph and pick a vertex $x \in D\left(H_{i}\right)$ such that $H_{i+1}=H_{i}-\{x\}$ is an induced, non-scattered subgraph of $H_{i}$. This method of obtaining an induced, non-scattered subgraph $H_{i+1}$ from $H_{i}$ by eliminating a vertex from the dominating set $D\left(H_{i}\right)$ is called the dominating vertex elimination method (DVE method) (see [2] for more details). Let $H$ be a simple finite and connected graph; then, the length of the maximum chain of induced, non-scattered, subgraphs of $H$ obtained by successively applying DVE method is called graphical degree stability and is denoted as $\operatorname{Stab}_{d}(H)$. In other words, if $H=H_{0}{ }^{H_{1}{ }^{H_{r}}}$ is the maximum chain of induced, non-scattered, subgraphs of $H$ obtained by using DVE method, then $\operatorname{Stab}_{d}(H)$. Note that the vertex set of $H_{j}$ consists of $[n-j]$ vertices where $1 \leq j \leq r$.

Definition 5. Let $H$ be a simple, finite, and connected graph with vertex set $V(H)=\left\{x_{1}, x_{2}, \ldots, x_{n}\right\}$ having degree sequence of vertices $\left(d_{1}, d_{2}, \ldots, d_{n}\right)$ such that $d_{1} \geq d_{2} \geq \cdots \geq d_{n}$ and $d_{i} \geq 0, \forall 1 \leq i \leq n$; then, the ideal $Q(H)=\left\langle x_{1}^{d_{1}}, x_{2}^{d_{2}}, \ldots, x_{n}^{d_{n}}\right\rangle$ is called the sequential ideal associated to the graph $H$. Let $H=H_{0}{ }^{H_{1}}{ }^{{ }^{H}{ }_{r}}$ be the maximum chain of induced, non-scattered, subgraphs of $H$ obtained by using the DVE method with $\operatorname{Stab}_{d}(H)=r$ and $Q\left(H_{i}\right)=\left\langle x_{1}^{d_{i_{1}}}, x_{2}^{\mathscr{S}_{i_{2}}}, \ldots, x_{n-i}^{d_{i_{n-i}}}\right\rangle$ be the sequential ideal corresponding to $H_{i}, 0 \leq i \leq r$; then, the elimination ideal of $H$ is defined as

$$
I_{D}(H)=Q_{H_{0}} \cap Q_{H_{1}} \cap \cdots \cap Q_{H_{r}} .
$$

Let $S=K\left[x_{1}, x_{2}, \ldots, x_{n}\right], n \geq 2$, be a polynomial ring over infinite field $K$ and $I \subseteq S$ be a monomial ideal of $S$. Let $G(I)$ be the minimal set of monomial generators of $I$ and the highest degree of monomial in $G(I)$ is denoted as $\operatorname{deg}(I)$. Also, let $I_{\geq t}$ be an ideal generated by monomial of $I$ of degree $\geq t$. Given a monomial $u \in S$, set $m(u)=\max \left\{i: x_{i} \mid u\right\}$ and $m(I)=\max _{u \in G(I)} m(u)$. A monomial ideal $I$ is stable if for any $u \in G(I)$ we have $x_{i} \cdot u / x_{m(u)} \in I$ for all $1 \leq i<m(u)$.

Eisenbud et al. proved the following result in [12].

Proposition 1. Let $I$ be a monomial ideal such that $I_{\geq e}$ is stable, where $e \geq \operatorname{deg}(I)$ is an integer. Then, $\operatorname{reg}(I) \leq e$.

Remark 1. Let $I$ and $J$ are two monomial ideals with $r \geq \operatorname{deg}(I)$ and $s \geq \operatorname{deg}(J)$, where $r$ and $s$ are two integers such that $I_{\geq r}$ and $J_{\geq s}$ are stable ideals; then, $(I \cap J)_{\geq \max \{r, s\}}$ is stable ideal.

\section{Regularity of $K_{n} \vee C_{m}$}

In this section, we present our results regarding the Castelnuovo-Mumford regularity of elimination ideals associated to join operation of complete graph and cyclic graph.

In [2], the authors gave the following formula to compute the graphical degree stability for cyclic graphs.

Proposition 2. If $C_{m}, m \geq 3$, is the cyclic graph, then

$$
\operatorname{Stab}_{d}\left(C_{m}\right)= \begin{cases}\frac{m}{3}, & \text { if } m \equiv 0(\bmod 3), \\ \frac{m-1}{3}, & \text { if } m \equiv 1(\bmod 3), \\ \frac{m-2}{3}, & \text { if } m \equiv 2(\bmod 3) .\end{cases}
$$

Lemma 1. Let $K_{n}, n \geq 2$, be a complete graph and $C_{m}, m \geq 4$, be a cyclic graph; then,

$$
\operatorname{Stab}_{d}\left(K_{n} \vee C_{m}\right)=n+\operatorname{Stab}_{d}\left(C_{m}\right) .
$$

Proof. We shall prove it by induction on $n$. Let $n=2$; then, $H_{0}=K_{2} \vee C_{m}$ with degree sequence

$$
(m+1, m+1, \underbrace{4, \ldots, 4}_{m \text {-tuple }}),
$$

and $D\left(H_{0}\right)=\left\{x_{1}, x_{2}\right\}$. Without loss of generality, pick $x_{1} \in D\left(H_{0}\right)$, and using the DVE method, we get $H_{1}$ with the degree sequence (always rearrange the sequence after applying DVE method $(m, \underbrace{3, \ldots, 3}_{m \text {-tuple }})$ and $D\left(H_{1}\right)=\left\{x_{1}\right\}$. Again applying the DVE method, we get $H_{2}=C_{m}$. So, 
$\operatorname{Stab}_{d}\left(K_{2} \vee C_{m}\right)=2+\operatorname{Stab}_{d}\left(C_{m}\right)$. Suppose that result is true for $n=p$; then, the degree sequence $K_{p} \vee C_{m}$ will be

$$
\begin{gathered}
(\underbrace{p+m-1, \ldots, p+m-1}_{p \text {-tuple }}, \underbrace{p+2, \ldots, p+2}_{m \text {-tuple }}), \\
\operatorname{Stab}_{d}\left(K_{p} \vee C_{m}\right)=p+\operatorname{Stab}_{d}\left(C_{m}\right) .
\end{gathered}
$$

Consider $n=p+1$; then, $H_{0}=K_{p+1} \vee C_{m}$ with degree sequence

$$
(\underbrace{p+m, \ldots, p+m}_{p+1 \text {-tuple }}, \underbrace{p+3, \ldots, p+3}_{m \text {-tuple }})
$$

and $\left|V\left(H_{0}\right)\right|=p+m+1$, with $D\left(H_{0}\right)=\left\{x_{1}, \ldots, x_{p+1}\right\}$ as $m \geq 4$ which are precisely the vertices that initially belonged to $K_{p+1}$. Without loss of generality, pick $x_{1} \in D\left(H_{0}\right)$, and applying the DVE method, we get $H_{1}=K_{p} \vee C_{m}$.

$$
\begin{aligned}
& \Rightarrow \operatorname{Stab}_{d}\left(K_{p+1} \vee C_{m}\right)=1+\operatorname{Stab}_{d}\left(K_{p} \vee C_{m}\right)=1+p+\operatorname{Stab}_{d}\left(C_{m}\right)=(1+p)+\operatorname{Stab}_{d}\left(C_{m}\right) \\
& \Rightarrow \operatorname{Stab}_{d}\left(K_{p+1} \vee C_{m}\right)=(p+1)+\operatorname{Stab}_{d}\left(C_{m}\right)
\end{aligned}
$$

Corollary 1. Let $K_{n}, n \geq 2$, be a complete graph and $C_{m}, m \geq 4$, be a cyclic graph; then, the sequential ideal of $K_{n} \vee C_{m}$ is given as

$$
Q_{i}= \begin{cases}\left\langle x_{1}^{n+m-i-1}, \ldots, x_{n-i}^{n+m-i-1}, x_{n-i+1}^{n-i+2}, \ldots, x_{n+m-i}^{n-i+2}\right\rangle, & 0 \leq i \leq n, \\ \left\langle x_{1}^{2}, \ldots, x_{m-3(i-n)}^{2}, x_{m-3(i-n)+1}, \ldots, x_{n+m-i}\right\rangle, & n+1 \leq i \leq n+\operatorname{Stab}_{d}\left(C_{m}\right) .\end{cases}
$$

Proof. This proof is immediate from Lemma 1.

Proposition 3. Let $K_{n} \vee C_{m}, n \geq 2$ and $m \geq 4$, be the join of complete and cyclic graphs, respectively, and

$$
Q_{i}=\left\langle x_{1}^{n+m-i-1}, \ldots, x_{n-i}^{n+m-i-1}, x_{n-i+1}^{n-i+2}, \ldots, x_{n+m-i}^{n-i+2}\right\rangle
$$

be the sequential ideal associated to $H_{i}$; then, $Q_{i}$ is stable ideal, where $\gamma(i)=(n-i)^{2}+m(2 n-2 i+1)+2(i-n)+1$ and $0 \leq i \leq n$.

Proof. Let $H_{0}=K_{n} \vee C_{m}, n \geq 2$ and $m \geq 4$, be the join of complete and cyclic graphs, respectively; then, by Corollary 1 , sequential ideal associated to $H_{i}, 0 \leq i \leq n$, is given as $Q_{i}=$ $\left\langle x_{1}^{a_{1}}, \ldots, x_{n+m-i}^{a_{n+m-i}}\right\rangle$ where

$$
a_{k}=\left\{\begin{array}{l}
n+m-i-1, \quad 1 \leq k \leq n-i, \\
n-i+2, \quad n-i+1 \leq k \leq n+m-i .
\end{array}\right.
$$

Let $\gamma(i)=(n-i)^{2}+m(2 n-2 i+1)+2(i-n)+1$, for all $0 \leq i \leq n$. We shall show that $Q_{i_{>\gamma(i)}}$ is a stable ideal. Take $u \in Q_{i_{>\gamma(i)}}$; then, $u=v x_{k}^{a_{k}}$ for some $1 \leq k \leq n+m-i$ where $v \in\left\langle x_{1}, \ldots, x_{n+m-i}\right\rangle^{\gamma(i)-a_{k}}$.

If $m(u)>k$, then $x_{l} u / x_{m(u)}=x_{l} v / x_{m(u)} x_{k}^{a_{k}} \in Q_{i_{z \gamma(i)}}$ for all $l<m(u)$. So, $Q_{i}$ is stable.

If $m(u)=k$, then clearly $u \in\left\langle x_{1}, \ldots, x_{n+m-i}\right\rangle^{\gamma^{(i)}}$.

$$
\Rightarrow Q_{i_{z \gamma(i)}} \subseteq\left\langle x_{1}, \ldots, x_{n+m-i}\right\rangle^{\gamma(i)} \text {. }
$$

We show that $\left\langle x_{1}, \ldots, x_{n+m-i}\right\rangle^{\gamma(i)} \subseteq Q_{i_{\geq \gamma(i)}}$. Let $w \in\left\langle x_{1}, \ldots, x_{n+m-i}\right\rangle^{\gamma(i)}$; then, $w=x_{1}^{\beta_{1}} x_{2}^{\beta_{2}}, \ldots, x_{n+m-i}^{\beta_{n+m-i}}$ with $\beta_{s} \geq 0$ for all $1 \leq s \leq n+m-i$ and $\sum_{s=1}^{n+m-i} \beta_{s} \geq \gamma(i)$. Therefore, there exists at least one $s \in\{1, \ldots, n+m-i\}$ such that $\beta_{s} \geq a_{s}$ and $w=\left(x_{1}^{\beta_{1}}, \ldots, x_{s}^{\beta_{s}-a_{s}}, \ldots, x_{n+m-i}^{\beta_{n+m-i}}\right) x_{s}^{a_{s}} \in Q_{i_{\geq \gamma(i)}}$, and the result follows.

Proposition 4. Let $K_{n} \vee C_{m}, n \geq 2$ and $m \geq 4$, be the join of complete and cyclic graphs, respectively, and

$$
Q_{i}=\left\langle x_{1}^{2}, \ldots, x_{m-3(i-n)}^{2}, x_{m-3(i-n)+1}, \ldots, x_{n+m-i}\right\rangle
$$

be the sequential ideal associated to $G_{i}, n+1 \leq i \leq n+\operatorname{Stab}_{d}\left(C_{m}\right)$; then, $Q_{i_{\geq \gamma(i)}}$ is stable ideal, where $\gamma(i)=m+3 n-3 i+1$.

Proof. Let $H_{0}=K_{n} \vee C_{m}, n \geq 2$ and $m \geq 4$, be the join of complete and cyclic graphs, respectively; then, by Corollary 1 , sequential ideal associated to $H_{i}, n+1 \leq i \leq$ $n+\operatorname{Stab}_{d}\left(C_{m}\right)$, is given as $Q_{i}=\left\langle x_{1}^{a_{1}}, \ldots, x_{n+m-i}^{a_{n+m-i}}\right\rangle$ where

$$
a_{k}= \begin{cases}2, & 1 \leq k \leq m-3(i-n), \\ 1, & m-3(i-n)+1 \leq k \leq n+m-i .\end{cases}
$$

Let $\quad \gamma(i)=m+3 n-3 i+1, \quad$ for all $n+1 \leq i \leq n+\operatorname{Stab}_{d}\left(C_{m}\right)$; then, by similar arguments as above, $Q_{i_{z \gamma(i)}}$ is a stable ideal.

Theorem 1. Let $K_{n}, n \geq 2$, be a complete graph and $C_{m}, m \geq 4$, be a cyclic graph. Then, $\operatorname{reg}\left(I_{D}\left(K_{n} \vee C_{m}\right)\right) \leq$ $n^{2}+m(2 n+1)-2 n+1$. 
Proof. If $\operatorname{Stab}_{d}\left(C_{m}\right)=p$, then by using Proposition 2, $\operatorname{Stab}_{d}\left(K_{n} \vee C_{m}\right)=n+p$, so the corresponding elimination ideal is given as $I_{D}\left(K_{n} \vee C_{m}\right)=\cap_{i=0}^{n+p} Q_{i}$, and by using Propositions 3 and 4 and Remark $1, I_{D}\left(K_{n} \vee C_{m}\right)$ is stable for $\gamma$, where

$$
\gamma=\max \{\gamma(i), \mid 0 \leq i \leq n+p\}=n^{2}+m(2 n+1)-2 n+1 .
$$

By using Proposition 1,

$$
\operatorname{reg}\left(I_{D}\left(K_{n} \vee C_{m}\right)\right) \leq n^{2}+m(2 n+1)-2 n+1 .
$$

Example 1. Consider $K_{2} \vee C_{4}$, where $n=2$ and $m=4$ (see Figure 1).

$$
\begin{aligned}
& D\left(H_{0}\right)=\left\{x_{1}, x_{2}\right\}, \quad Q_{0}=\left\langle x_{1}^{5}, x_{2}^{5}, x_{3}^{4}, x_{4}^{4}, x_{5}^{4}, x_{6}^{4}\right\rangle, \quad \text { and } \\
& \operatorname{reg}\left(Q_{0}\right)=21 . \\
& D\left(H_{1}\right)=\left\{x_{1}\right\}, \quad Q_{1}=\left\langle x_{1}^{4}, x_{2}^{3}, x_{3}^{3}, x_{4}^{3}, x_{5}^{3}\right\rangle, \quad \text { and } \\
& \operatorname{reg}\left(Q_{1}\right)=12 . \\
& D\left(H_{2}\right)=\left\{x_{1}, x_{2}, x_{3}, x_{4}\right\}, \quad Q_{2}=\left\langle x_{1}^{2}, x_{2}^{2}, x_{3}^{2}, x_{4}^{2}\right\rangle, \quad \text { and } \\
& \operatorname{reg}\left(Q_{2}\right)=5 . \\
& D\left(H_{3}\right)=\left\{x_{1}\right\}, Q_{3}=\left\langle x_{1}^{2}, x_{2}, x_{3}\right\rangle, \text { and } \operatorname{reg}\left(Q_{3}\right)=2 .
\end{aligned}
$$

Remark 2. In general, $Q_{i_{>\gamma(i)-1}}$ is not stable, where $Q_{i}$ are sequential ideals of $K_{n} \vee C_{m}^{2 \gamma(i)-1}$, where $0 \leq i \leq n+\operatorname{Stab}_{d}\left(C_{m}\right)$. As in Example 1, $Q_{1_{\geq 11}}$ is not a stable ideal for $i=1$ and $\gamma(1)=12$.

\section{Regularity of $K_{n} * C_{m}$}

In this section, we present our results concerning the Castelnuovo-Mumford regularity of elimination ideals associated to corona product operation of complete graph and cyclic graphs.

Lemma 2. Let $K_{n}, n \geq 2$, be a complete graph and $C_{m}, m \geq 4$, be a cyclic graph; then,

$$
\operatorname{Stab}_{d}\left(K_{n} * C_{m}\right)=n+n \operatorname{Stab}_{d}\left(C_{m}\right)
$$

Proof. We shall prove it by induction on $n$. Let $n=2$; then, $H_{0}=K_{2} * C_{m}$ with degree sequence

$$
(m+1, m+1, \underbrace{3, \ldots, 3}_{m \text {-tuple }}, \underbrace{3, \ldots, 3}_{m-\text { tuple }}),
$$

and $D\left(H_{0}\right)=\left\{x_{1}, x_{2}\right\}$. Without loss of generality, pick $x_{1} \in D\left(H_{0}\right)$, and using the DVE method, we get $H_{1}$ with the degree sequence

$$
(m, \underbrace{3, \ldots, 3}_{m \text {-tuple }}, \underbrace{2, \ldots, 2}_{m \text {-tuple }}) .
$$

Now $D\left(H_{1}\right)=\left\{x_{1}\right\}$, and again applying the DVE method, we have only two copies of $\mathrm{C}_{m}$ in $\mathrm{H}_{2}$ with degree sequence

$$
\begin{array}{r}
(\underbrace{2, \ldots, 2}_{m-\text { tuple }}, \underbrace{2, \ldots, 2}_{m-\text { tuple }}), \\
\Rightarrow \operatorname{Stab}_{d}\left(K_{2} * C_{m}\right)=2+2 \operatorname{Stab}_{d}\left(C_{m}\right) .
\end{array}
$$

Suppose that result is true for $n=p$; then, the degree sequence of $K_{p} * C_{m}$ will be

$$
\begin{array}{r}
(\underbrace{p+m-1, \ldots, p+m-1}_{p \text {-tuple }}, \underbrace{\underbrace{3, \ldots, 3}_{m-\text { tuple }}, \ldots, \underbrace{3, \ldots, 3}_{m-\text { tuple }}}_{\text {pm-tuple }}) \\
\Rightarrow \operatorname{Stab}_{d}\left(K_{p} * C_{m}\right)=p+p \operatorname{Stab}_{d}\left(C_{m}\right) .
\end{array}
$$

Consider $n=p+1$; then, $H_{0}=K_{p+1} * C_{m}$ with degree sequence

$$
(\underbrace{p+m, \ldots, p+m}_{p+1-\text { tuple }}, \underbrace{3, \ldots, 3}_{m(p+1)-\text { tuple }}, \ldots, \underbrace{3, \ldots, 3}_{m-\text { tuple }}),
$$

and $\left|V\left(H_{0}\right)\right|=p m+p+m+1$ with $D\left(H_{0}\right)=\left\{x_{1}, \ldots\right.$, $\left.x_{p+1}\right\}$ which are precisely the vertices that initially belonged to $K_{p+1}$ because $m \geq 4$. By applying the DVE method, we get $K_{p} * C_{m}$ and one copy of $C_{m}$, and we call this graph $H_{1}$.

$$
\begin{aligned}
& \Rightarrow \operatorname{Stab}_{d}\left(K_{p+1} * C_{m}\right)=1+\operatorname{Stab}_{d}\left(K_{p} * C_{m}\right)+\operatorname{Stab}_{d}\left(C_{m}\right)=1+p+p \operatorname{Stab}_{d}\left(C_{m}\right)+\operatorname{Stab}_{d}\left(C_{m}\right), \\
& \Rightarrow \operatorname{Stab}_{d}\left(K_{p+1} * C_{m}\right)=(p+1)+(p+1) \operatorname{Stab}_{d}\left(C_{m}\right) .
\end{aligned}
$$



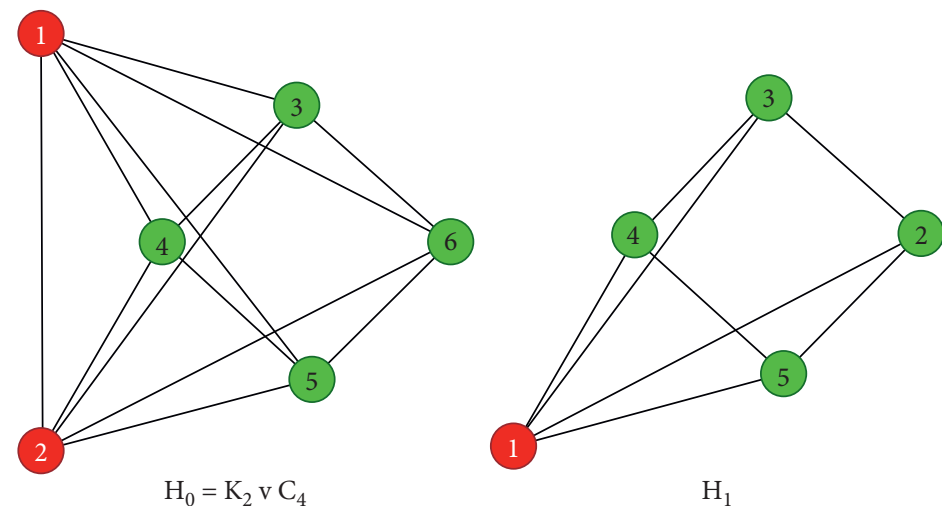

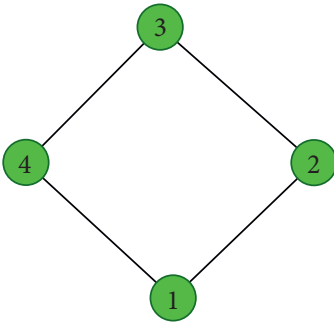

$\mathrm{H}_{2}$

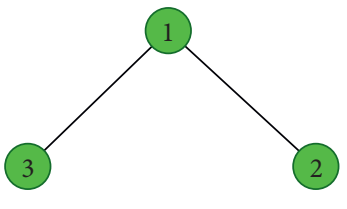

$\mathrm{H}_{3}$

Figure 1: $H_{0}, H_{1}, H_{2}, H_{3}$.

Corollary 2. Let $K_{n}, n \geq 2$, be a complete graph and $C_{m}, m \geq 4$, be a cyclic graph; then, the sequential ideal of $K_{n} * C_{m}$ is given as

$$
Q_{i}= \begin{cases}\left\langle x_{1}^{n+m-1}, \ldots, x_{n}^{n+m-1}, x_{n+1}^{3}, \ldots, x_{r}^{3}\right\rangle, & i=0, \\ \left\langle x_{1}^{n+m-i-1}, \ldots, x_{n-i}^{n+m-i-1}, x_{n-i+1}^{3}, \ldots, x_{r-i(m+1)}^{3}, x_{r-i(m+1)+1}^{2}, \ldots, x_{r-i}^{2}\right\rangle, & 1 \leq i \leq n-1, \\ \left\langle x_{1}^{2}, \ldots, x_{n m}^{2}\right\rangle, & i=n, \\ \left\langle x_{1}^{2}, \ldots, x_{n m-3(i-n)}^{2}, x_{n m-3(i-n)+1}, \ldots, x_{r-i}\right\rangle, & n+1 \leq i \leq n+n \operatorname{Stab}_{d}\left(C_{m}\right),\end{cases}
$$

where $r=n+n m$

Proof. The proof is immediate from Lemma 2.

Proposition 5. Let $K_{n} * C_{m}, n \geq 2$ and $m \geq 4$, be the corona product of complete and cyclic graphs, respectively, and

$$
Q_{0}=\left\langle x_{1}^{n+m-1}, \ldots, x_{n}^{n+m-1}, x_{n+1}^{3}, \ldots, x_{r}^{3}\right\rangle,
$$

be the sequential ideal associated to $H_{0}$; then, $Q_{0_{x y}(0)}$ is stable ideal, where $r=n+n m$ and $\gamma(0)=n^{2}+n(3 m-2)+1$.

Proof. Let $H_{0}=K_{n} * C_{m}, n \geq 2$ and $m \geq 4$, be the corona product of complete and cyclic graphs, respectively; then, by Corollary 2, the sequential ideal associated to $H_{0}$ is given as $Q_{0}=\left\langle x_{1}^{a_{1}}, \ldots, x_{r}^{a_{r}}\right\rangle$ where

$$
a_{j}= \begin{cases}n+m-1, & 1 \leq j \leq n, \\ 3, & n+1 \leq j \leq r .\end{cases}
$$

Let $\gamma(0)=n^{2}+n(3 m-2)+1$. We show that $Q_{0_{y \gamma(0)}}$ is a stable ideal. Take $u \in Q_{0_{z \gamma(0)}}$; then, $u=v x_{k}^{a_{k}}$ for some $1 \leq k \leq r$ where $v \in\left(x_{1}, \ldots, x_{r}\right)^{\gamma(0)-a_{k}}$.

If $m(u)>k$, then $x_{l} u / x_{m(u)}=\left(x_{l} v / x_{m(u)}\right) x_{k}^{a_{k}} \in Q_{0_{z \gamma(0)}}$ for all $l<m(u)$. So, $Q_{0_{z \gamma(0)}}$ is stable.

If $m(u)=k$, then clearly $u \in\left\langle x_{1}, \ldots, x_{r}\right\rangle^{\gamma(0)}$.

$$
\Rightarrow Q_{0_{z \gamma(0)}} \subseteq\left\langle x_{1}, \ldots, x_{r}\right\rangle^{\gamma(0)} \text {. }
$$

We show that $\left\langle x_{1}, \ldots, x_{r}\right\rangle^{\gamma(0)} \subseteq Q_{0_{z \gamma(0)}}$. Let $w \in\left\langle x_{1}, \ldots, x_{r}\right\rangle^{\gamma(0)}$; then, $w=x_{1}^{\beta_{1}} x_{2}^{\beta_{2}} \cdots x_{r}^{\beta_{r}}$ with $\beta_{s} \geq 0$ for all $1 \leq s \leq r$ and $\sum_{s=1}^{r} \beta_{s} \geq \gamma(0)$. Therefore, there exists at least one $s \in\{1, \ldots, r\}$ such that $\beta_{s} \geq a_{s}$ and $w=\left\langle x_{1}^{\beta_{1}}, \ldots, x_{s}^{\beta_{s}-a_{s}}, \ldots, x_{r}^{\beta_{r}}\right\rangle x_{s}^{a_{s}} \in Q_{0_{z \gamma(0)}}$, and the result follows.

Proposition 6. Let $K_{n} * C_{m}, n \geq 2$ and $m \geq 4$, be the corona product of complete and cyclic graphs, respectively, and

$$
Q_{i}=\left\langle x_{1}^{n+m-i-1}, \ldots, x_{n-i}^{n+m-i-1}, x_{n-i+1}^{3}, \ldots, x_{r-i(m+1)}^{3}, x_{r-i(m+1)+1}^{2}, \ldots, x_{r-i}^{2}\right\rangle,
$$

be the sequential ideal associated to $H_{i}$; then, $Q_{i_{2 \gamma(i)}}$ is stable ideal, where $r=n+n m, \gamma(i)=(n-i)^{2}-2(n+m i-i)+$ $3 n m+1$ and $1 \leq i \leq n-1$.
Proof. Let $H_{0}=K_{n} * C_{m}, n \geq 2$ and $m \geq 4$, be the corona product of complete and cyclic graphs, respectively; then, by Corollary 2, sequential ideal associated to $H_{i}, 1 \leq i \leq n-1$, is given as $Q_{i}=\left\langle x_{1}^{a_{1}}, \ldots, x_{r-i}^{a_{r-i}}\right\rangle$ where 


$$
a_{j}= \begin{cases}n+m-i-1, & 1 \leq j \leq n-i, \\ 3, & n-i+1 \leq j \leq r-i(m+1), \\ 2, & r-i(m+1)+1 \leq j \leq r-i .\end{cases}
$$

Let $\gamma(i)=(n-i)^{2}-2(n+m i-i)+3 n m+1$, for all $1 \leq i \leq n-1$. We shall show that $Q_{i_{\geq \gamma(i)}}$ is a stable ideal. Take $u \in Q_{i_{>\gamma(i)}}$; then, $u=v x_{k}^{a_{k}}$ for some $1 \leq k \leq r-i$ where $v \in\left(x_{1}, \ldots, x_{r-i}\right)^{\gamma(i)-a_{k}}$.

If $m(u)>k$, then $x_{l} u / x_{m(u)}=x_{l} v / x_{m(u)} x_{k}^{a_{k}} \in Q_{i_{z \gamma(i)}}$ for all $l<m(u)$. So, $Q_{i_{>\gamma}(i)}$ is stable.

If $m(u)=k$, then clearly $u \in\left\langle x_{1}, \ldots, x_{r-i}\right\rangle^{\gamma^{(i)}}$.

$$
\Rightarrow Q_{i_{\geq \gamma(i)}} \subseteq\left\langle x_{1}, \ldots, x_{r-i}\right\rangle^{\gamma(i)} .
$$

We show that $\left\langle x_{1}, \ldots, x_{r-i}\right\rangle^{\gamma(i)} \subseteq Q_{i_{\geq \gamma(i)}}$. Let $w \in\left\langle x_{1}, \ldots, x_{r-i}\right\rangle^{\gamma(i)}$; then, $w=x_{1}^{\beta_{1}} x_{2}^{\beta_{2}}, \ldots, x_{r-i}^{\beta_{r-i}}$ with $\beta_{s} \geq 0$ for all $1 \leq s \leq r-i$ and $\sum_{s=1}^{r-i} \beta_{s} \geq \gamma(i)$. Therefore, there exists at least one $s \in\{1, \ldots, r-i\}$ such that $\beta_{s} \geq a_{s}$ and $w=\left\langle x_{1}^{\beta_{1}}, \ldots, x_{s}^{\beta_{s}-a_{s}}, \ldots, x_{r-i}^{\beta_{r-i}}\right\rangle x_{s}^{a_{s}} \in Q_{i_{\geq \gamma(i)}}$, and the result follows.

Proposition 7. Let $K_{n} * C_{m}, n \geq 2$ and $m \geq 4$, be the corona product of complete and cyclic graphs, respectively, and $Q_{n}=$ $\left\langle x_{1}^{2}, \ldots, x_{n m}^{2}\right\rangle$ be the sequential ideal associated to $H_{n}$; then, $Q_{n_{z \gamma(n)}}$ is stable ideal, where $r=n+n m$ and $\gamma(n)=n m+1$.

Proof. Let $K_{n} * C_{m}, n \geq 2$ and $m \geq 4$, be the corona product of complete and cyclic graphs, respectively; then, by Corollary 2, sequential ideal associated to $H_{n}$ is given as $Q_{n}=\left\langle x_{1}^{2}, \ldots, x_{n m}^{2}\right\rangle$. Let $\gamma(n)=n m+1$; then, by similar arguments as above, $Q_{n_{\geq \gamma(n)}}$ is a stable ideal.

Proposition 8. Let $K_{n} * C_{m}, n \geq 2$ and $m \geq 4$, be the corona product of complete and cyclic graphs, respectively, and

$$
Q_{i}=\left\langle x_{1}^{2}, \ldots, x_{n m-3(i-n)}^{2}, x_{n m-3(i-n)+1}, \ldots, x_{r-i}\right\rangle,
$$

be the sequential ideal associated to $H_{i}$; then, $Q_{i_{>\gamma(i)}}$ is stable ideal, where $r=n+n m, \gamma(i)=n m+3(n-i))^{+(i)}+1$, and $n+1 \leq i \leq n+n \operatorname{Stab}_{d}\left(C_{m}\right)$.

Proof. Let $K_{n} * C_{m}, n \geq 2$ and $m \geq 4$, be the corona product of complete and cyclic graphs, respectively; then, by Corollary 2 , sequential ideal associated to $H_{i}, n+1 \leq i \leq$ $n+n \operatorname{Stab}_{d}\left(C_{m}\right)$, is given as $Q_{i}=\left\langle x_{1}^{a_{1}}, \ldots, x_{r-i}^{a_{r-i}}\right\rangle$, where

$$
a_{j}= \begin{cases}2, & 1 \leq j \leq n m-3(i-n), \\ 1, & n m-3(i-n)+1 \leq j \leq r-i .\end{cases}
$$

Let $\gamma(i)=n m+3(n-i)+1$

for all $n+1 \leq i \leq n+n \operatorname{Stab}_{d}\left(C_{m}\right)$. We shall show that $Q_{i_{z \gamma(i)}}$ is a stable ideal. Take $u \in Q_{i_{>\gamma(i)}}$; then, $u=v x_{k}^{a_{k}}$ for some $1 \leq k \leq r-i$ where $v \in\left\langle x_{1}, \ldots, x_{r-i}\right\rangle^{\gamma(i)-a_{k}}$.
If $m(u)>k$, then $x_{l} u / x_{m(u)}=\left(x_{l} v / x_{m(u)}\right) x_{k}^{a_{k}} \in Q_{i_{\geq \gamma(i)}}$ for all $l<m(u)$. So, $Q_{i_{\geq \gamma(i)}}$ is stable.

If $m(u)=k$, then clearly $u \in\left\langle x_{1}, \ldots, x_{r-i}\right\rangle^{\gamma(i)}$.

$$
\Rightarrow Q_{i_{\geq \gamma(i)}} \subseteq\left\langle x_{1}, \ldots, x_{r-i}\right\rangle^{\gamma(i)} \text {. }
$$

We show that $\left\langle x_{1}, \ldots, x_{r-i}\right\rangle^{\gamma(i)} \subseteq Q_{i_{>\gamma(i)}}$. Let $w \in\left\langle x_{1}, \ldots, x_{r-i}\right\rangle^{\gamma(i)}$; then, $w=x_{1}^{\beta_{1}} x_{2}^{\beta_{2}}, \ldots, x_{r-i}^{\beta_{r-i}}$ with $\beta_{s} \geq 0$ for all $1 \leq s \leq r-i$ and $\sum_{s=1}^{r-j} \beta_{s} \geq \gamma(i)$. Therefore, there exists at least one $s \in\{1, \ldots, r-i\}$ such that $\beta_{s} \geq a_{s}$ and $w=\left\langle x_{1}^{\beta_{1}}, \ldots, x_{s}^{\beta_{s}-a_{s}}, \ldots, x_{r-i}^{\beta_{r-i}}\right\rangle x_{s}^{a_{s}} \in Q_{i_{\geq \gamma(i)}}$, and the result follows.

Theorem 2. Let $K_{n}, n \geq 2$, be a complete graph and $C_{m}, m \geq 4$, be a cyclic graph; then, we have

$$
\operatorname{reg}\left(I_{D}\left(K_{n} * C_{m}\right)\right) \leq n^{2}+n(3 m-2)+1 .
$$

Proof. If $\operatorname{Stab}_{d}\left(C_{m}\right)=p$, then by using Proposition 2, $\operatorname{Stab}_{d}\left(K_{n} * C_{m}\right)=n+n p$, so the corresponding elimination ideal is given as $I_{D}\left(K_{n} * C_{m}\right)=\cap_{i=0}^{n+n p} Q_{i}$, and by using Propositions 5-8 and Remark $1, I_{D}\left(K_{n} * C_{m}\right)$ is stable for $\gamma$, where

$$
\gamma=\max \{\gamma(i) \mid 0 \leq i \leq n+n p\}=n^{2}+n(3 m-2)+1 .
$$

By using Proposition 1,

$$
\operatorname{reg}\left(I_{D}\left(K_{n} * C_{m}\right)\right) \leq n^{2}+n(3 m-2)+1
$$

Example 2. Consider $K_{2} * C_{4}$, where $n=2$ and $m=4$ (see Figure 2).

$$
\begin{aligned}
& D\left(H_{0}\right)=\left\{x_{1}, x_{2}\right\}, \\
& Q_{0}=\left\langle x_{1}^{5}, x_{2}^{5}, x_{3}^{3}, x_{4}^{3}, x_{5}^{3}, x_{6}^{3}, x_{7}^{3}, x_{8}^{3}, x_{9}^{3}, x_{10}^{3}\right\rangle, \\
& \operatorname{reg}\left(Q_{0}\right)=25 . \\
& D\left(H_{1}\right)=\left\{x_{1}\right\}, Q_{1}=\left\langle x_{1}^{4}, x_{2}^{3}, x_{3}^{3}, x_{4}^{3}, x_{5}^{3}, x_{6}^{2}, x_{7}^{2}, x_{8}^{2}, x_{9}^{2}\right\rangle, \\
& \text { and } \operatorname{reg}\left(Q_{1}\right)=16 . \\
& D\left(H_{2}\right)=\left\{x_{1}, x_{2}, x_{3}, x_{4}, x_{5}, x_{6}, x_{7}, x_{8}\right\}, \\
& Q_{2}=\left\langle x_{1}^{2}, x_{2}^{2}, x_{3}^{2}, x_{4}^{2}, x_{5}^{2}, x_{6}^{2}, x_{7}^{2}, x_{8}^{2}\right\rangle, \text { and } \operatorname{reg}\left(Q_{2}\right)=9 . \\
& D\left(H_{3}\right)=\left\{x_{1}, x_{2}, x_{3}, x_{4}, x_{5}\right\}, \\
& Q_{3}=\left\langle x_{1}^{2}, x_{2}^{2}, x_{3}^{2}, x_{4}^{2}, x_{5}^{2}, x_{6}, x_{7}\right\rangle, \text { and } \operatorname{reg}\left(Q_{3}\right)=6 . \\
& D\left(H_{4}\right)=\left\{x_{1}, x_{2}\right\}, \quad Q_{4}=\left\langle x_{1}^{2}, x_{2}^{2}, x_{3}, x_{4}, x_{5}, x_{6}\right\rangle, \quad \text { and } \\
& \operatorname{reg}\left(Q_{4}\right)=3 .
\end{aligned}
$$

Remark 3. In general, one cannot get $Q_{i_{\geq y(i)-1}}$ stable, where $Q_{i}$ are the sequential ideals of $K_{n} * C_{m}, 0 \leq i \leq n+n \operatorname{Stab}_{d}\left(C_{m}\right)$. As in Example 2, for $i=2 \gamma(2)=9$ and $Q_{2>8}$ is not a stable ideal.

Remark 4. Elimination ideals are Borel type ideals, and upper bound for Borel type ideals was discussed in $[3,13]$; it 


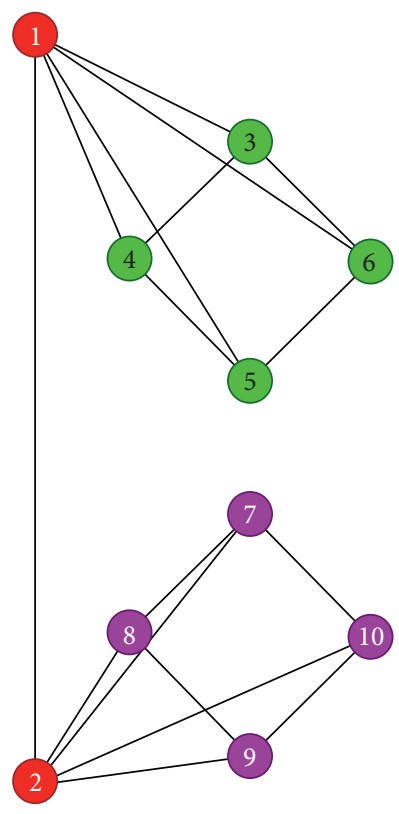

$\mathrm{H}_{0}$
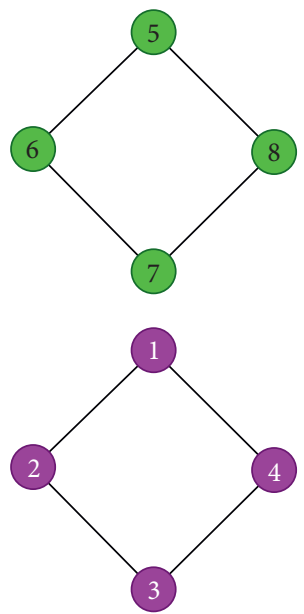

$\mathrm{H}_{2}$
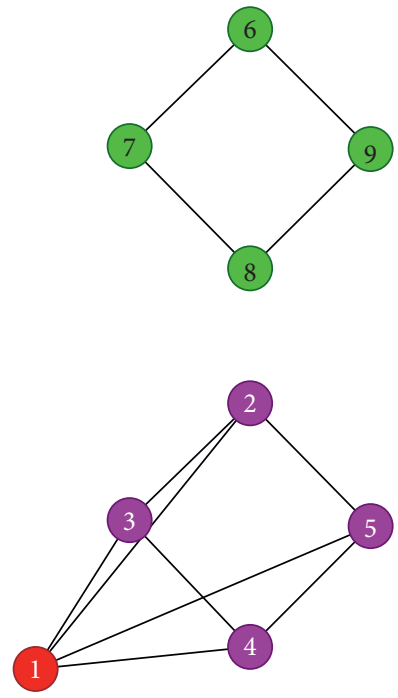

$\mathrm{H}_{1}$
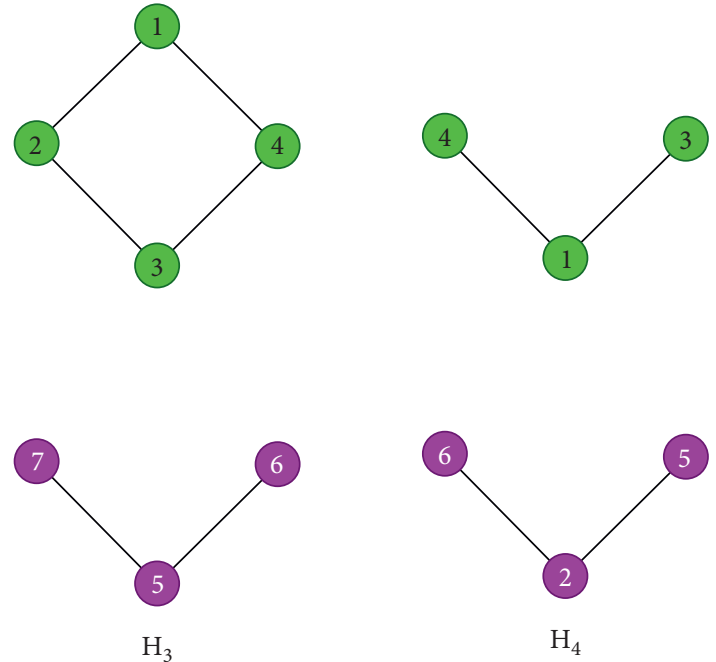

Figure 2: $H_{0}, H_{1}, H_{2}, H_{3}, H_{4}$.

is worthy to note that our given bounds are sharper than the ones given in $[3,13]$.

\section{Data Availability}

The data used to support the findings of this study are included within this article.

\section{Conflicts of Interest}

The authors declare that they have no conflicts of interest.

\section{Authors' Contributions}

Zongming Lv verified and analyzed the results and arranged the funding for this study. Muhammad Junaid Ali Junjua proved the results. Muhammad Tajammal Tahir wrote the first version of the paper. Khurram Shabbir proposed the problem and supervised this work.

\section{Acknowledgments}

This study was supported by the Higher Education Commission (Islamabad) through the National Research Program for Universities (grant no. 7359/Punjab/NRPU/R\&D/ HEC/2017).

\section{References}

[1] D. Bayer and M. Stillman, "A criterion for detectingm-regularity," Inventiones Mathematicae, vol. 87, no. 1, pp. 1-11, 1987.

[2] I. Anwar and A. Khalid, "Algebraic characterization of graphical degree stability," Communications of the Korean Mathematical Society, vol. 34, no. 1, pp. 113-125, 2019. 
[3] S. Ahmad and I. Anwar, "An upper bound for the regularity of ideals of borel type," Communications in Algebra, vol. 36, no. 2, pp. 670-673, 2008.

[4] D. Bayer and D. Mumford, "What can be computed in algebraic geometry?" in Proceedings of the Computational Algebraic Geometry and Commutative Algebra, Symposia Mathematica XXXIV, pp. 1-48, Cambridge, UK, 1993.

[5] B. Bollobas, "Degree sequences of random graphs," Discrete Mathematics, vol. 33, pp. 1-19, 1981.

[6] R. I. Tyshkevich, A. A. Chernyak, and Zh. A. Chernyak, "Graphs and degree sequences I," Cybernetics, vol. 23, no. 6, pp. 734-745, 1987.

[7] S. L. Hakimi, "On realizability of a set of integers as degrees of the vertices of a linear graph. I," Journal of the Society for Industrial and Applied Mathematics, vol. 10, no. 3, pp. 496506, 1962.

[8] M. J. A. Junjua, K. Shabbir, and A. Naseem, "Regularity of elimination ideals of certain family of graphs, to be appear in ars combinatoria," Preprint.

[9] I. M. Adamu, "Homomorphism of intuitionistic fuzzy multigroups," Open Journal of Mathematical Sciences, vol. 4, no. 1, pp. 430-441, 2020.

[10] P. A. Ejegwa, M. A. Ibrahim, and M. A. Ibrahim, "On divisible and pure multigroups and their properties," Open Journal of Mathematical Sciences, vol. 4, no. 1, pp. 377-385, 2020.

[11] A. Douhadji, Y. Awussi, and Y. Awussi, "Characterization of a vector measure: application in the $\backslash(\mathrm{GL}(2 ; \mid \operatorname{mathbb}\{\mathrm{R}\}) \backslash)$ group," Open Journal of Mathematical Sciences, vol. 4, no. 1, pp. 74-77, 2020.

[12] D. Eisenbud, A. Reeves, and B. Totaro, "Initial ideals of veronese subrings," Advances in Mathematics, vol. 109, pp. 168-187, 1995.

[13] M. Cimpoeaş, "A stable property of Borel types ideals," Communications in Algebra, vol. 36, no. 2, pp. 674-677, 2008. 